\title{
Study on Precision Polishing Technology Combining Electrophoresis and Magnetic Finishing
}

\author{
Lieh-Dai Yang', Kun-Ling Wu ${ }^{2}$,, Chin-Chang Yeh ${ }^{3}$, Hsin-Min Lee ${ }^{4}$ \\ ${ }^{1}$ Department of Industrial Management, Nankai University of Technology, Nantou, Taiwan \\ ${ }^{2}$ Department of Mechanical Engineering, Tungnan University, New Taipei City, Taiwan \\ ${ }^{3}$ Department of Mechanical Engineering, Oriental Institute of Technology, New Taipei City, Taiwan \\ ${ }^{4}$ Mechanical Engineering Department, Army Academy, Taoyuan City, Taiwan
}

\section{Email address:}

t215@nkut.edu.tw (Lieh-Dai Yang), klwu@mail.tnu.edu.tw (Kun-Ling Wu), ccyeh@mail.oit.edu.tw (Chin-Chang Yeh), 1sm5762@gmail.com (Hsin-Min Lee)

${ }^{*}$ Corresponding author

\section{To cite this article:}

Lieh-Dai Yang, Kun-Ling Wu, Chin-Chang Yeh, Hsin-Min Lee. Study on Precision Polishing Technology Combining Electrophoresis and Magnetic Finishing. International Journal of Materials Science and Applications. Vol. 5, No. 6, 2016, pp. 235-240.

doi: $10.11648 /$ j.ijmsa.20160506.11

Received: October 6, 2016; Accepted: October 15, 2016; Published: October 17, 2016

\begin{abstract}
The aim of this paper is to improve the quality of surface roughness of precision molds, which is difficult to control in the traditional polishing technology, and to provide a kind of equipment which is simple and easy to operate in order to obtain a high precision in surface finishing and polishing. The combination of magnetic force polishing and electrophoretic polishing is mainly to combine the magnetic grinding mechanism with the electrophoretic deposition method to conduct finishing and polishing experiments. From the experimental results we can see that the combination of magnetic force and electrophoretic polishing can make the original roughness of the discharge surface of $1.7 \mu \mathrm{m}$ down to $0.08 \mu \mathrm{m}$ which is mirror effect after improving for 20 minutes. The experimental results show that the precision polishing combined with electrophoresis and magnetic grinding can effectively improve the surface roughness and therefore reduce technical and personnel training costs, shorten the production cycle time, and thus improve production efficiency. It can be concluded as a quite competitive technology in the industry.
\end{abstract}

Keywords: EDM, Magnetic Abrasive, Electrophoretic Deposition, Surface Roughness

\section{Introduction}

With the ever-changing technology, high-performance products in the request, the new materials are developed out unceasingly. These high-tech materials inheres with high strength, hardness, toughness, heat resistance and corrosion resistance and other excellent mechanical properties. For the surface with the nature and dimensional accuracy of the product, the use of cutting and grinding and other traditional processing method is very difficult to work, the main reason is that the traditional method used in the direct contact with the workpiece is easy to produce high stress problems and leadto poor processing quality. Therefore, the use of electrical, thermal, chemical, mechanical and other non-traditional processing came into being. However, non-contact electrical discharge machining is the most widely used. It is often widely used in precision molds industry, but the discharge surface roughness cannot meet the needs of the industry, so a variety of polishing are required to achieve the request [1-3]. The traditional polishing method, although has its effect to improve the surface, but it is also difficult to control the precise quality of the product. Therefore, Shinmura et al. used permanent magnets to investigate the effects of magnetic flux density, vibration frequency and other parameters on the surface roughness and material removal of stainless steel and tool steels $[4,5]$. Shinmura et al. investigated the effect of particle size of magnetic abrasive grains on the surface roughness and material removal of SK4 tool steels [6]. Kremen et al. applied themechanical analysis of material removal and established a mathematical model of true 
circularity error for magnetic abrasive grinding [7]. Khairy first deduced the mathematical formula of grinding pressure and magnetic flux density, and then experimentally studied the effect of grinding pressure on surface roughness and observed the removal effect of edge burrs [8]. Jain et al. used an intermittent magnetic field to investigate the effect of work gap and workpiece speed on surface roughness and material removal [9]. Kim used WC-Co magnetic abrasives for SUS304 tubes to investigate workpiece speed, abrasive fluid content and vibration frequency on the surface roughness [10, 11]. Takahata et al. used micro-EDM to fabricate the microstructure, and then to use $\mathrm{Al}_{2} \mathrm{O}_{3}$ particles adsorbing on the electrode in the $100 \mu \mathrm{m}$ diameter, the workpiece to move back and forth $430 \mu \mathrm{m}$ distance way to do polishing [12]. Y. Tani et al. used electrophoretic deposition of silicon particles to polish silicon wafers and to process the glass [13]. Chen et al. developed a screw polishing process that is the use of a high-speed screw-driven abrasive polishing of the workpiece surface technology [14-16]. After the study of the former scholars, this paper will combine electrophoresis and magnetic grinding of precision polishing technology after discharge processing, to provide equipment with simple and easy to operate composite polishing technology and effectively improve the work piece surface roughness.

\section{Experimental Methods}

\subsection{Electrophoretic Deposition Principle}

General electrophoretic deposition method can be divided into constant voltage and constant current. In the constant voltage process, the potential difference of the suspension will decrease with the thickness of the deposited layer. The decrease of the potential difference of the suspension is liable to cause the colloidal particles to deposit at different rates. In the constant current process, since the constant current mode can provide a stable potential difference, all the colloidal particles in the deposition process are a fixed speed to the opposite polarity of the electrode movement, making the deposition rate easy to maintain a fixed value. In the electrophoretic deposition process, a constant voltage mode will be chosen to use because the constant voltage method is easier to deposit than to use the constant current mode, as shown in Figure 1.

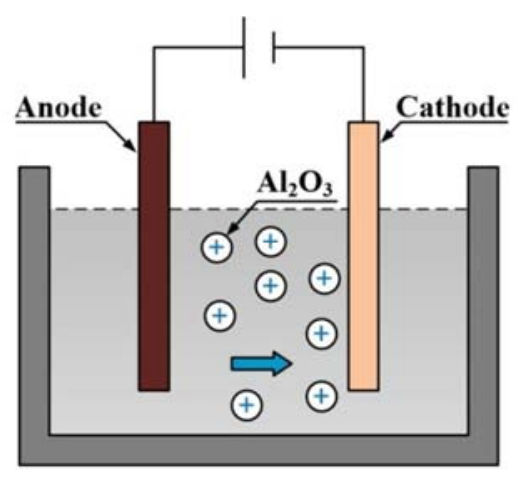

Figure 1. Schematic diagram of electrophoresis.

\subsection{Experimental Equipment}

In this experiment, using $\mathrm{CNC}$ milling machine as the main experimental equipment, by its high stability of the characteristics of the workpiece grinding and polishing. Because of the electrophoretic deposition mechanism required a stable position, so with a processing slot to place the workpiece. In order to make the polishing effect can be more significant in a relatively short period of time, so the load applied to the workpiece and the pressure bearing system was also built under the processing tank, the amount of compression by the spring then converted to the actual load on the workpiece. Figure 2 shows the electrophoretic deposition mechanism and experimental schematic diagram.

\subsection{Experimental Materials}

In this experiment, the workpiece material used was SKD61. The main components contained $0.42 \mathrm{wt} \% \mathrm{C}, 1.2 \mathrm{wt} \% \mathrm{Si}, 0.5$ $\mathrm{wt} \% \mathrm{Mn}, 5.5 \mathrm{wt} \% \mathrm{Cr}, 1.6 \mathrm{wt} \%$ Mo and $1.1 \mathrm{wt} \% \mathrm{v}$. The size of the workpiece is $40 \times 40 \times 6 \mathrm{~mm}$.

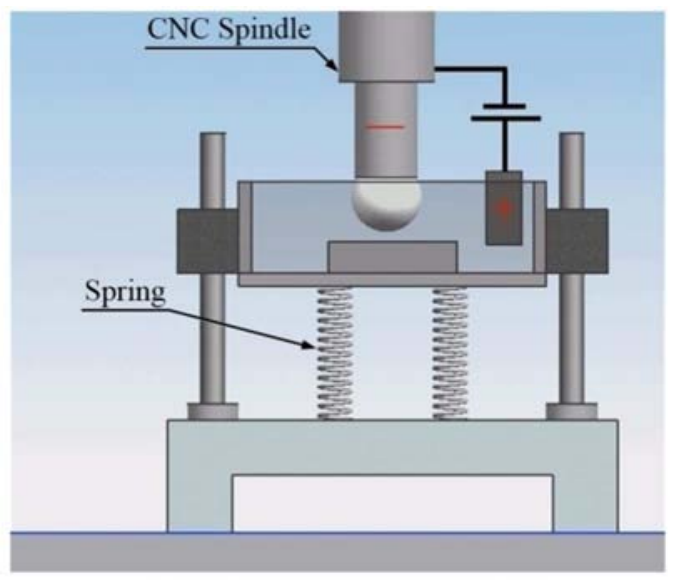

Figure 2. Schematic diagram of the test equipment.

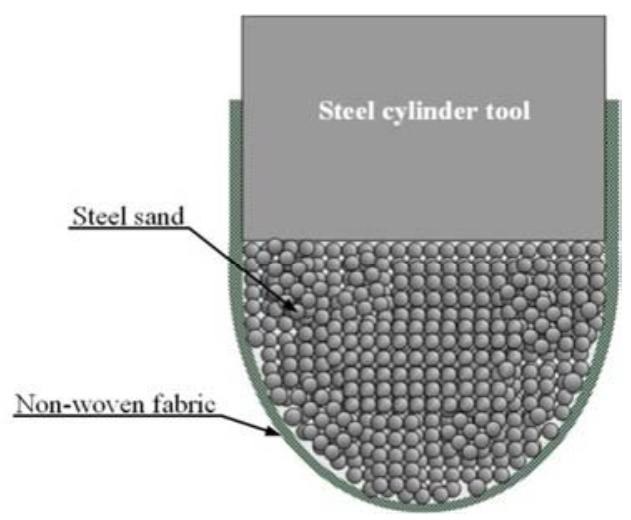

Figure 3. Schematic diagram of magnetic pole coating.

\subsection{Experimental Procedures}

Figure 4 showed the experimental procedures. Details as follow:

(1) The making of the discharge surface: first to grind using SKD61 tool steel material $40 \times 40 \times 6$ mmand then to conduct EDM to reach surface roughness $(\mathrm{Ra})$ with $1.7 \mu \mathrm{m}$. 
(2) The preparation of electrophoresis solution: first choose the best adsorption parameters of modulation solution, then to place $800 \mathrm{cc}$ pure water in a beaker, and then add the desired concentration of $\mathrm{Al}_{2} \mathrm{O}_{3}$ powder weight, to stir using a magnetic stirrer and then to drop HClin order to obtain $\mathrm{pH}$ value 4 . At this point, the process still needed to stir more than 90 minutes until the $\mathrm{pH}$ value is stable.

(3) The coated magnetic pole: after magnetic pole adsorption of iron powder, the iron powder will be coated using non-woven fabric. At this point, the role of non-woven iron powder is to provide a deformable elastomer, and when the magnetic pole on the electricity, the iron powder also plays the role of the electrode, making $\mathrm{Al}_{2} \mathrm{O}_{3}$ deposition toward its adsorption, and non-woven fabric function is to contain the particles move to avoid polishing off, increasing the power of abrasive cutting. As shown in Figure 3.

(4) The polishing: the workpiece fixed in the self-make processing tank and injecting $\mathrm{Al}_{2} \mathrm{O}_{3}$ solution into the tank, and then set parameters to grind and finish the SKD61 tool steel. Table1 shows the experimental parameters.

(5) The cleaning and measuring workpiece: the processed specimen with the acetone soaked will be put into ultrasonic cleaning machine for 20 minutes after cleaning to be dry and to avoid rust, and then surface roughness will be measured by roughness tester.

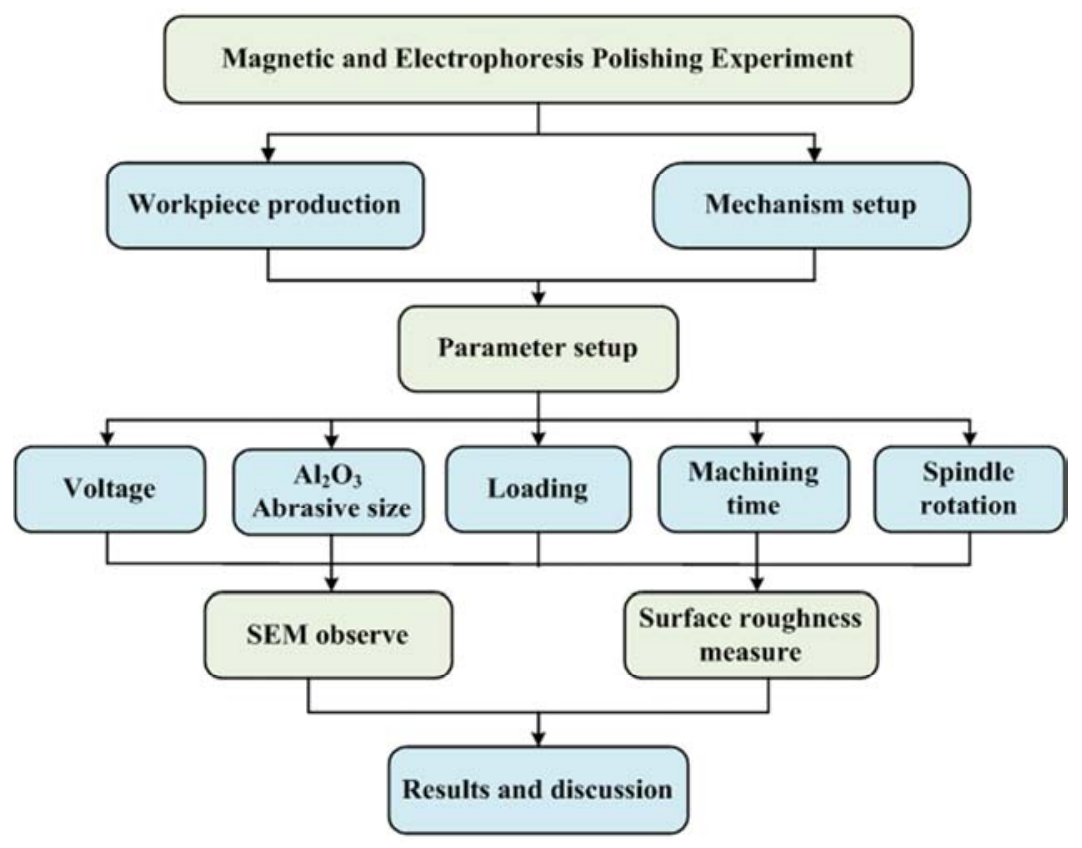

Figure 4. Experimental flow chart.

Table 1. Preferred parameter combinations.

\begin{tabular}{ll}
\hline $\mathrm{Al}_{2} \mathrm{O}_{3}$ Abrasive size $(\mu \mathrm{m})$ & $1,0.3$ \\
Concentration (wt $\%)$ & 12 \\
Spindle rotation (rpm) & $100,200,300$, \\
& $400,500,600$ \\
Loading (g) & $100,150,200$, \\
Voltage (V) & 250,300 \\
Machining time (min) & $10,20,30,40$ \\
\hline
\end{tabular}

\section{Results and Discussion}

\subsection{Preliminary Experiment}

In order to verify the effect of the electrophoretic deposition mechanism, the same processing parameters were used in the absence of power supply, so that the $\mathrm{Al}_{2} \mathrm{O}_{3}$ particles could not be deposited and adsorbed in order to conduct pure abrasive polishing, the results are shown in Fig. 5 It can be seen from Fig. 5 that under the same processing conditions, the presence or absence of the electrophoretic deposition mechanism is significantly different from the experimental results. Particles due to electrophoresis effect and gathered in the end face, making the region of the polishing phenomenon is extremely obvious. The results showed that the surface roughness of the specimen was significantly higher than that of the non-woven fabric, and the surface roughness of the specimen was significantly higher than that of the non-woven fabric.

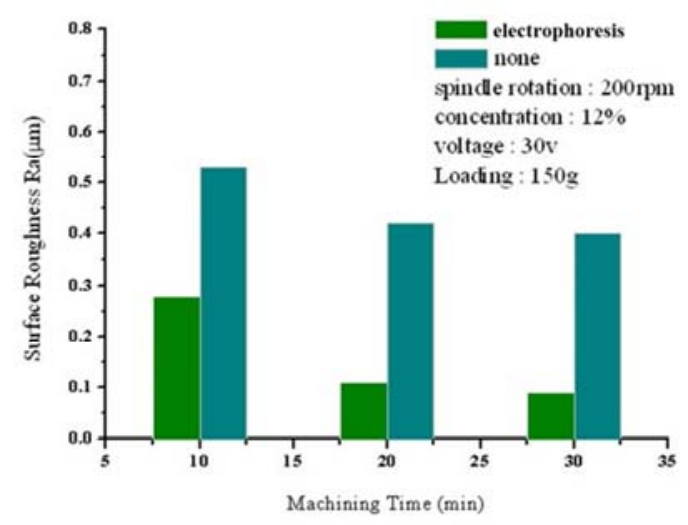

Figure 5. Effect of adding electrophoresis mechanism on polishing. 


\subsection{The Effectof Processing Time on Surface Roughness}

Time is one of the most important parameters in the mechanism of the combination of magnetic force and electrophoretic polishing. It can be found from Fig. 6 that the roughness can be reduced from $1.7 \mu \mathrm{m} \mathrm{Ra}$ to $0.28 \mu \mathrm{m}(\mathrm{Ra})$ at $10 \mathrm{~min}$. With the increase of the time, the roughness can be lowered to $0.1 \mu \mathrm{m}(\mathrm{Ra})$ at 20 minutes and the roughness value of $0.09 \mu \mathrm{m}(\mathrm{Ra})$ can be achieved at 30 minutes.

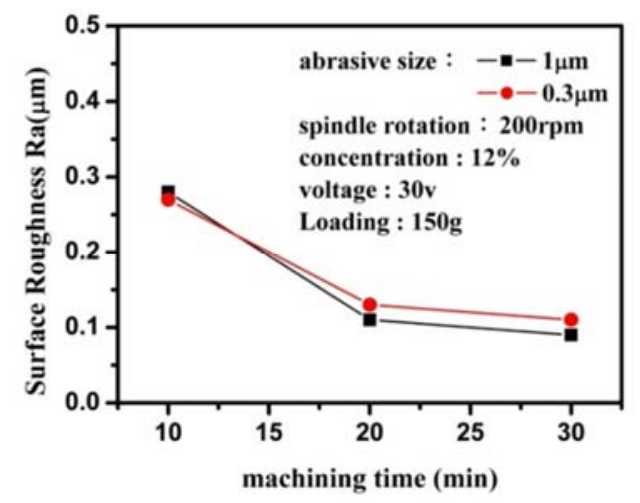

Figure 6. Effect of processing time on surface roughness

\subsection{The Effectof Spindle Speed on Surface Roughness}

The effect of spindle speed and centrifugal force on the adsorption capacity is significant.With the increase of speed will increase the centrifugal force and make the number of particles adsorbed to the electrode surface reduced, then the deposition is relatively lower. As shown in Fig.7, as the spindle speed increases, the roughness gradually improves, but after more than $300 \mathrm{rpm}$, as the spindle speed increases, the centrifugal force increases and the $\mathrm{Al}_{2} \mathrm{O}_{3}$ particles are not easily adsorbed and therefore surface roughness deteriorated.

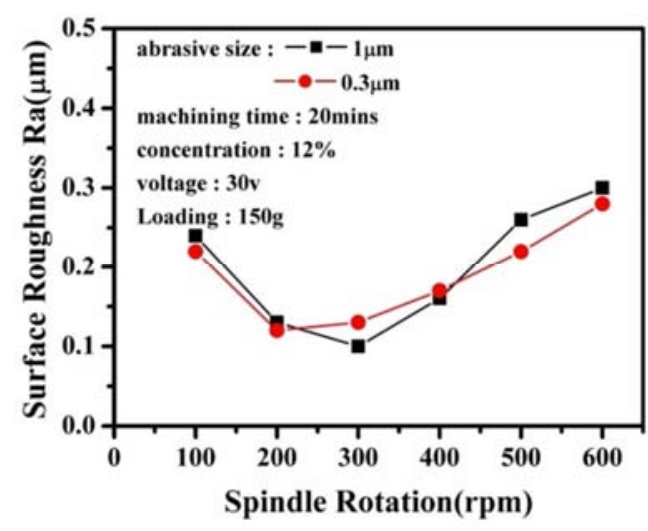

Figure 7. Effect of spindle speed on surface roughness.

\subsection{The Effect of Loading on Surface Roughness}

In order to achieve a polishing effect in a short time, an axial load is applied to the surface of the test piece. And this load is applied to the outer layer coated with iron powder between the non-woven fabric and test pieces and simultaneously considering the relevant processing parameters to explore the impact of surface roughness. Figure
8 showed the relationship between the load and roughness when performing electromagnetically coupled deposition and polishing. The SKD61 specimen was polished using different $\mathrm{Al}_{2} \mathrm{O}_{3}$ particle sizes $(1 \mu \mathrm{m}, 0.3 \mu \mathrm{m})$. It can be seen from Figure 8 , when the other parameters are fixed and the load is $150 \mathrm{~g}$, the effect of grinding and polishing is the best, using $1 \mu \mathrm{m}$ and $0.3 \mu \mathrm{m}$ its surface roughness can reach $0.1 \mu \mathrm{m}(\mathrm{Ra}), 0.08 \mu \mathrm{m}$ (Ra). However, to increase the load, the test piece surface will be damaged and roughness will be worse significantly.

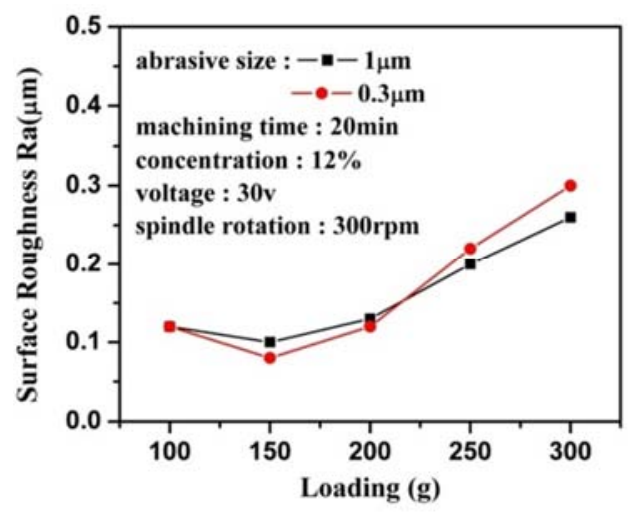

Figure 8. The loading effect on the surface roughness.

\subsection{The Effectof Voltage on Surface Roughness}

Electrophoretic deposition polishing is the application of electric field in the solution, resulting in electrophoretic effect of the particles moving to direction of the cathode iron powder adsorption. When the voltage increases, not only the deposition rate will be accelerated, and the adsorption of $\mathrm{Al}_{2} \mathrm{O}_{3}$ particles relative increase in the use of non-woven fabric constraints, making the polishing effect is more significant. As shown in Figure 9, when the voltage exceeds $30 \mathrm{~V}$, the surface roughness curve slowed down; simultaneously the use of $1 \mu \mathrm{m}$ particle size of the abrasive, the original roughness of $1.7 \mu \mathrm{m}(\mathrm{Ra})$ can be reduced to $0.11 \mu \mathrm{m}(\mathrm{Ra})$.

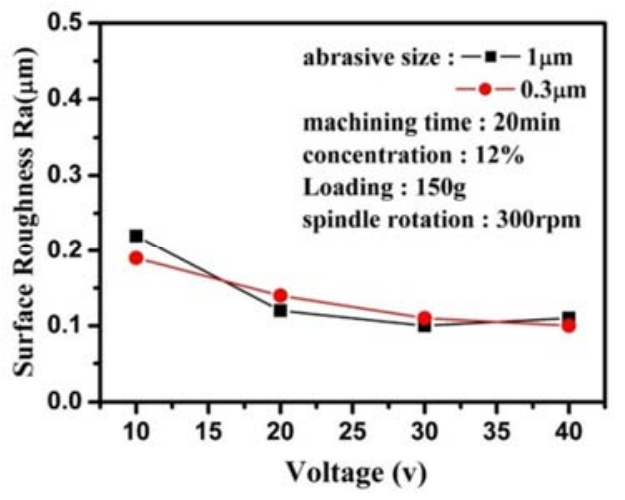

Figure 9. Effect of voltage on surface roughness.

\subsection{Optimal Combination of Parameters and Surface Observation}

Table 2 showed that the optimal combination of process parameters obtained from the above single-factor experiments. With this set of parameters, the surface roughness of the test 
piece can be reduced to $0.08 \mu \mathrm{m}(\mathrm{Ra})$. Figure 10indicated that roughness value measured by the roughness tester. Figure 11 showed the surface profile observation of the test piece and the SEM results using 3D profiler. Itcan be found that polished surface is smooth and flat. The polished surface has been near mirroreffect shown in Figure12.

Table 2. Preferred parameter combinations.

\begin{tabular}{ll|ll}
\hline $\mathrm{Al}_{2} \mathrm{O}_{3}$ size & $1 \mu \mathrm{m}(12 \%)$ & Voltage & $40(\mathrm{~V})$ \\
Spindle rotation & $300(\mathrm{rpm})$ & Machining time & $20(\mathrm{~min})$ \\
Loading $(\mathrm{g})$ & $150(\mathrm{~g})$ & Surface roughness $\mathrm{Ra}$ & $0.08(\mu \mathrm{m})$ \\
\hline
\end{tabular}

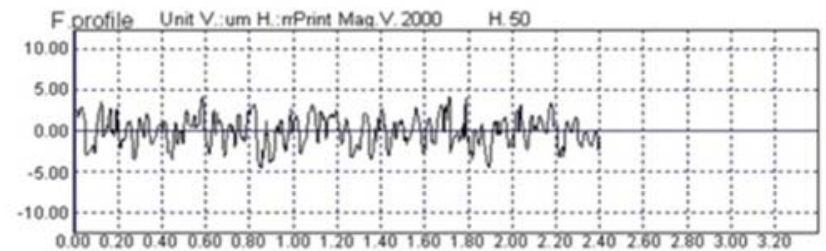

(a)

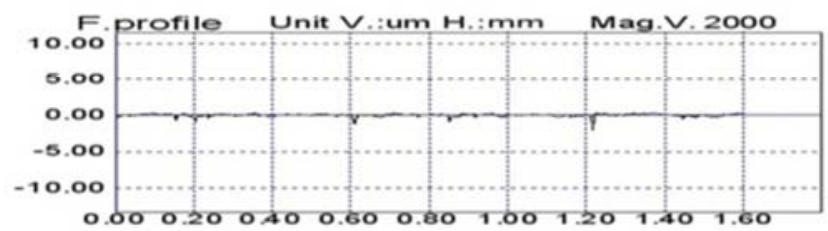

(b)

Figure 10. (a) Original surface roughness $1.7 \mu m$ (Ra)(b) Surface after processing $0.08 \mu \mathrm{m}(\mathrm{R} a)$
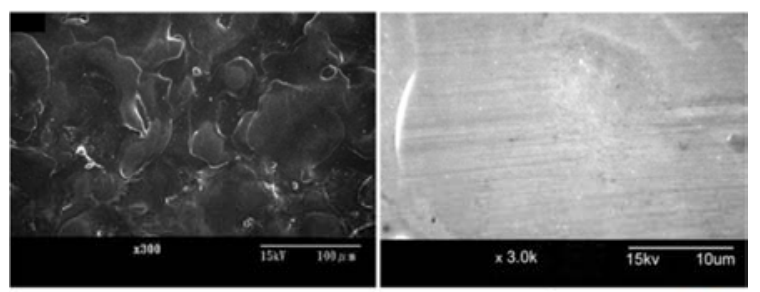

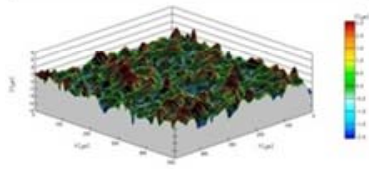

(a)

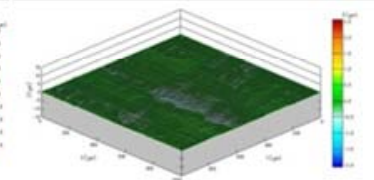

(b)
Figure 11. (a) EDM surface SEM and $3 D$ contours(b) SEM and $3 D$ profile of the polished surface.

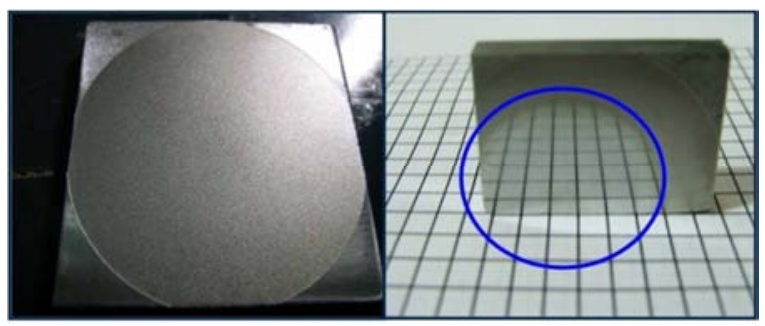

Figure 12. (a) EDM surface (b) The degree of the mirror surface after polishing.

\subsection{Use the Preferred Parameters to Polish the Surface}

From the experimental results we can see that the combination of magnetic force and electrophoretic polishing can effectively process the flat test piece. Then, in order to verify the adaptability of the iron powder and non-woven fabric to the surface shape of the test piece, the 3D curve tank will be processed, the steps are as follows:

(1) The making of electrodes: first using CNC milling machine for processing electrolytic copper to get the desired electrode shape.

(2) The making of curve groove test specimen: employing EDM to produce the required surface groove, according to the set current to obtain the test piece surface roughness of $1.7 \mu \mathrm{m}$ (Ra).

(3) The polishing: using the better combination of process parameters for processing test piece; the iron powder can be changed with the shape of test piece to fit surface of the groove and to achieve the effect of polishing, the roughness can reach $0.18 \mu \mathrm{m}(\mathrm{Ra})$, as shown in Figure 13.

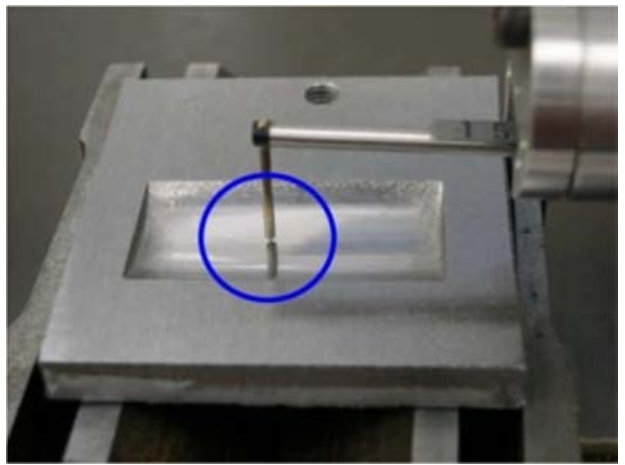

Figure 13. Surface roughness after processing up to $0.18 \mu m(R a)$.

\section{Conclusion}

The results of the study are summarized as follows:

(1) The magnetic force combined with electrophoretic deposition to test the polishing process, can effectively improve the grinding efficiency and the surface quality.

(2) The preferred polishing parameters would be $\mathrm{Al} 2 \mathrm{O} 3$ abrasive particle size of $1 \mu \mathrm{m}$, the concentration of $12 \%$, speed $300 \mathrm{rpm}$, load $150 \mathrm{~g}$, voltage $40 \mathrm{v}$, time 20 minutes.

(3) The preferred parameters obtained after the experiments were applied to conduct plane grinding and polishing, the results showed that the original discharge surface $1.7 \mu \mathrm{m}(\mathrm{Ra})$ can be down to $0.08 \mu \mathrm{m}$ (Ra) after 20 minutes.

(4) When polishing 3D curve groove, because the iron powder can withstand the deformation of the elastic body, it can fit the surface shape changes of the test piece, and then to do polishing with the experimental parameters, the surface roughness can also be decreased from $1.7 \mu \mathrm{m}(\mathrm{Ra})$ to $0.18 \mu \mathrm{m}$ (Ra). The effect of 3D polishing is also significant.

\section{Acknowledgements}

The authors would like to thank the Ministry of Science and Technology of the Republic of China, Taiwan, for financially supporting this research under Contract No. NSC MOST 104-2221-E-236 -005. 


\section{References}

[1] H. P. Tsui, B.H. Yan, W. T. Wu, and S. T. Hsu, A study on stainless steel mirror surface polishing by using the electrophoretic deposition method, International Journal of Machine Tools and Manufacture, Vol.47, No.12-13, 2007, pp. $1965-1970$

[2] L. Besra, M. Liu, A review on fundamentals and applications of electrophoretic deposition (EPD), Progress in Materials Science, Vol. 52,No. 1, 2007,pp.1-61

[3] P. Saraeian, H. S. Mehr, B. Moradi, H. Tavakoli,and O. K. Alrahmani, Study of Magnetic Abrasive Finishing for AISI321 Stainless Stee,1, Materials and Manufacturing Processes, 2016, pp. 2023-2029

[4] T. Shinmura, Study on plane magnetic abrasive finishing (3rd report) - on the finishing characteristics of non-ferromagnetic substances, J. ofJSPE, Vol. 55, No. 7, 1989, pp. 1271-1276

[5] T. Shinmura, Development of a unit system magnetic abrasive finishing apparatus using permanent magnets, Bull. Japan Soc. Of Prec. Engg., Vol. 23, No. 4, 1989, pp. 313-315

[6] T. Shinmura, K. Takazawa, E. Hatano, and M. Matsunaga, Study on magnetic abrasive finishing, Annals of the CIRP, Vol. 39, No. 1, 1990, pp. 325-328

[7] G. Z. Kremen, E. A. Elsayed, and V. I. Rafalovich, Mechanism of material removal in the magnetic abrasive process and the accuracy of machining, International Journal of Production Research, Vol. 34, No. 9, 1996, pp. 2629-2638

[8] A. B. Khairy, Aspects of surface and edge finish by magneto abrasive particles, Journal of Materials Processing Technology, Vol. 116, 2001, pp. 77-83
[9] V. K. Jain, P. Kumar, P. K. Behera, and S. C. Jayswal, Effect of working gap and circumferential speed on the performance of magnetic abrasive finishing process, Wear, 250, 2001, pp. 384-390

[10] J. D. Kim, Polishing of ultra-clean inner surfaces using magnetic force, International Journal of Advanced Manufacturing Technology, Vol. 21, 2003, pp. 91-97

[11] V. C. Shukla, P. M. Pandey, Experimental investigations into sintering of magnetic abrasive powder for ultrasonic assisted magnetic abrasive finishing process, Materials and Manufacturing Processes, 2016, pp. 1-7

[12] K. Takahata, S. Aoki, and T.Sato, Fine Surface Finishing Method for 3-Dimensional Micro Structures, IEEE MEMS, 1996, pp. $73-78$

[13] Y. Tani, T. Saeki, Y. Samitsu, K. Kobayashi, and Y. Sato, Infeed Grinding of Silicon Wafers Applying Electrophoretic Deposition of Ultrafine Abrasives, Annals of the CIRP, Japan,1998。

[14] M. Das, V. K. Jain, and P. S. Ghoshdastidar, Nano finishing of flat workpieces using rotational-magnetorheological abrasive flow finishing (R-MRAFF) process, The International Journal of Advanced Manufacturing Technology, Vol.62, 2012, pp. $405-420$

[15] W. C. Chen, K. L. Wu, and B. H. Yan A study on the application of newly developed magneto-elastic abrasive to improving the surface roughness of the bore, International Journal of Advanced Manufacturing Technology,Vol.73, 2014, PP.1557-1566

[16] P. Kala and P. M. Pandey, Comparison of finishing characteristics of two paramagnetic materials using double disc magnetic abrasive finishing, Journal of Manufacturing Processes, Vol.17, 2015,pp.63-77 